\title{
Correction to: Prognostic Indicators of Persistent Post-Concussive Symptoms after Deployment-Related Mild Traumatic Brain Injury: A Prospective Longitudinal Study in U.S. Army Soldiers; DOI: 10.1089/neu.2015.4320
}

\footnotetext{
After publication of the article entitled, "Prognostic Indicators of Persistent Post-Concussive Symptoms after Deployment-Related Mild Traumatic Brain Injury: A Prospective Longitudinal Study in U.S. Army Soldiers,' by Stein MB et al., J. Neurotrauma 33, 2125-2132, it came to the authors' attention that this report contains an error. The error relates to how one of the independent variables was described in the Methods section. The error does not impact the study findings, but the description of the variable has been corrected.

The first paragraph of the Measures subsection has been corrected as follows: "For purposes of the analyses described here, any predeployment reporting of probable TBI with loss of consciousness of any duration was counted as being positive for probable TBI history before the index deployment.'

This correction resolves the error because any subsequent mentions of pre-deployment TBI reflect that definition. Also, the phrase "with LOC" has been added in three places:

1. Results, paragraph 2, sentence 2 now reads "Approximately one-third of soldiers reported having sustained TBI(s) with LOC before the index deployment $(34.0 \%$; $\mathrm{SE}=1.0 \%)$."

2. The label for row 4 in Table 1 now reads "Any lifetime pre-deployment TBI with LOC."

3. The label for row 4 in Table 2 now reads "Any lifetime pre-deployment TBI with LOC."

The online version of the article has been corrected to reflect these changes.

The authors regret this error.
} 